\title{
Digital appendix 3. Extended fiction syndicated by Tillotson's Fiction Bureau and published in nineteenth-century Australian newspapers
}

Most data relating to Tillotson's Fiction Bureau in this table are derived from Law, Serialising, using titles and syndication dates in his tables "3.3 Expensive Tillotson's serials not appearing in the Bolton Journal in the 1890s" (89), "A.2 Serial fiction in the Bolton Weekly Journal, 1871-89” (222-227), “A.3 Serial fiction in the Age, 1872-99” (229-231), “A.4 Syndication of the novels of M. E. Braddon” (232-237), "A.5 Syndication of the Novels of Wilkie Collins" (238-240) and information within the text on Farjeon's "No. 119 Great Porter Square” (75-76). The exception is for authors preceded by "\%”, for which information is drawn from Turner.

NB. While Law refers to the date of serialization in Britain, Turner identifies contract dates. This difference explains why the duration between the dates of British and Australian publication is greater for titles identified by Turner.

NB. A number of the below titles also appeared in other Australian newspapers at later dates. But I have only listed instances within a year or two of the Tillotson's syndication date.

* Early titles identified by Law where Tillotson's did not arrange colonial publication \$ Fiction classified by Law as "expensive"

\# Title also published in Express and Telegraph (digitized by Trove since harvest completed)

! Publication identified by Johnson-Woods, Index

^ Publication identified by Law, Serialising 


\begin{tabular}{|c|c|c|c|c|}
\hline Author & Title & $\begin{array}{l}\text { Tillotson's } \\
\text { Date }\end{array}$ & Australian Newspaper/s & $\begin{array}{l}\text { Australian } \\
\text { Date }\end{array}$ \\
\hline $\begin{array}{l}* \text { M. E. } \\
\text { Braddon }\end{array}$ & "Taken at the Flood" & 1873 & $\begin{array}{l}\text { \# Leader; South Australian } \\
\text { Chronicle }\end{array}$ & 1873 \\
\hline * Dora Russell & $\begin{array}{l}\text { "Footprints in the } \\
\text { Snow" }\end{array}$ & 1876 & $\begin{array}{l}\text { Maitland Mercury } \\
\text { South Australian Chronicle }\end{array}$ & $\begin{array}{l}1877 \\
1878\end{array}$ \\
\hline $\begin{array}{l}* \text { M. E. } \\
\text { Braddon }\end{array}$ & "An Open Verdict" & 1877 & $\begin{array}{l}\text { Leader; South Australian } \\
\text { Chronicle }\end{array}$ & 1877 \\
\hline $\begin{array}{l}\text { * Florence } \\
\text { Marryat }\end{array}$ & "Her Father's Name" & 1876 & Newcastle Morning Herald & 1878 \\
\hline $\begin{array}{l}* \text { M. E. } \\
\text { Braddon }\end{array}$ & "The Cloven Foot" & 1878 & $\begin{array}{l}\text { ! Age } \\
\text { \# Australian Town and } \\
\text { Country Journal; South } \\
\text { Australian Chronicle }\end{array}$ & $\begin{array}{l}1878 \\
1879\end{array}$ \\
\hline Dora Russell & "Beneath the Wave" & 1878 & $\begin{array}{l}\wedge \text { Australian Journal; South } \\
\text { Australian Chronicle }\end{array}$ & 1878 \\
\hline $\begin{array}{l}\text { Geo. Manville } \\
\text { Fenn }\end{array}$ & "Hard to Win" & 1879 & South Australian Chronicle & 1879 \\
\hline B. L. Farjeon & $\begin{array}{l}\text { "No. } 119 \text { Great Porter } \\
\text { Square" }\end{array}$ & 1879 & $\wedge$ Australian Journal & 1881 \\
\hline M. E. Braddon & "Just as I Am" & 1880 & $\begin{array}{l}\text { ! Age; South Australian } \\
\text { Chronicle }\end{array}$ & 1880 \\
\hline Dora Russell & "Quite True" & 1880 & South Australian Chronicle & 1880 \\
\hline $\begin{array}{l}\text { Eliza Lynn } \\
\text { Linton }\end{array}$ & "My Love" & 1880 & $\begin{array}{l}\text { Telegraph; Week } \\
\text { Australian Town and Country } \\
\text { Journal; ! Age }\end{array}$ & $\begin{array}{l}1880 \\
1881\end{array}$ \\
\hline Wilkie Collins & "Jezebel's Daughter" & 1880 & $\begin{array}{l}\text { Cootamundra Herald; } \\
\text { Goulburn Herald }\end{array}$ & 1882 \\
\hline $\begin{array}{l}\text { \% Ina L. } \\
\text { Cassilis }\end{array}$ & $\begin{array}{l}\text { "The Pale Lady of } \\
\text { Treherne" }\end{array}$ & 1880 & Fremantle Herald & 1880 \\
\hline John Saunders & "Victor or Victim?" & 1881 & Adelaide Observer; ! Age & 1881 \\
\hline $\begin{array}{l}\text { Robert } \\
\text { Buchanan }\end{array}$ & $\begin{array}{l}\text { "The Martyrdom of } \\
\text { Madeline" }\end{array}$ & 1881 & South Australian Chronicle & 1882 \\
\hline Dora Russell & "Croesus' Widow" & 1882 & South Australian Chronicle & 1882 \\
\hline Hawley Smart & "At Fault" & 1882 & $\begin{array}{l}\text { \# Evening News; South } \\
\text { Australian Chronicle }\end{array}$ & 1883 \\
\hline $\begin{array}{l}\text { W. Clark } \\
\text { Russell }\end{array}$ & "A Sea Queen" & 1882 & $\begin{array}{l}\text { Age; South Australian } \\
\text { Chronicle }\end{array}$ & 1883 \\
\hline $\begin{array}{l}\text { Margaret } \\
\text { Oliphant }\end{array}$ & "Sir Tom" & 1883 & $\begin{array}{l}\text { Adelaide Observer; Evening } \\
\text { Journal } \\
\text { Evening News }\end{array}$ & $\begin{array}{l}1883 \\
1884 \\
\end{array}$ \\
\hline M. E. Braddon & "Phantom Fortune" & 1883 & $\begin{array}{l}\text { \# Illustrated Sydney News; } \\
\text { South Australian Chronicle }\end{array}$ & 1883 \\
\hline $\begin{array}{l}\text { Adeline } \\
\text { Sergeant }\end{array}$ & "Jacobi's Wife" & 1883 & $\begin{array}{l}\text { Adelaide Observer; Evening } \\
\text { Journal }\end{array}$ & 1883 \\
\hline B. L. Farjeon & "The Sacred Nugget" & 1883 & $\begin{array}{l}\text { Adelaide Observer; Evening } \\
\text { Journal }\end{array}$ & 1884 \\
\hline Charles Reade & "Love and Money" & 1884 & $\begin{array}{l}\text { Adelaide Observer; Evening } \\
\text { Journal } \\
\text { Capricornian; Morning } \\
\text { Bulletin }\end{array}$ & $\begin{array}{l}1884 \\
1885\end{array}$ \\
\hline M. E. Braddon & "Ishmael" & 1884 & $\begin{array}{l}\text { Australian Town and Country } \\
\text { Journal; Age; Queenslander }\end{array}$ & 1884 \\
\hline M. E. Braddon & "Wyllard's Weird" & 1884 & $\begin{array}{l}\text { Leader } \\
\text { \# South Australian Chronicle }\end{array}$ & $\begin{array}{l}1884 \\
1885 \\
\end{array}$ \\
\hline $\begin{array}{l}\text { Compton } \\
\text { Reade }\end{array}$ & "Under Which King?" & 1884 & South Australian Chronicle & 1885 \\
\hline Hawley Smart & "Tie and Trick" & 1884 & South Australian Chronicle & 1885 \\
\hline Dora Russell & “James Daunton's & 1885 & Telegraph; Week & 1887 \\
\hline
\end{tabular}




\begin{tabular}{|c|c|c|c|c|}
\hline & Fate" & & & \\
\hline $\begin{array}{l}\text { Justin } \\
\text { McCarthy }\end{array}$ & "Camiola" & 1885 & $\begin{array}{l}\text { South Australian Chronicle; } \\
\text { Sydney Mail }\end{array}$ & 1885 \\
\hline $\begin{array}{l}\text { \% William } \\
\text { Black }\end{array}$ & $\begin{array}{l}\text { "The Wise Women of } \\
\text { Inverness" }\end{array}$ & 1885 & South Australian Chronicle & 1885 \\
\hline $\begin{array}{l}\text { Geo. Manville } \\
\text { Fenn }\end{array}$ & $\begin{array}{l}\text { "The Master of the } \\
\text { Ceremonies" }\end{array}$ & 1885 & Age; Narracoorte Herald & 1885 \\
\hline M. E. Braddon & "Cut by the Country" & 1885 & South Australian Chronicle & 1885 \\
\hline B. L. Farjeon & "Aunt Parker" & 1885 & Queenslander & 1885 \\
\hline Wilkie Collins & "The Evil Genius" & 1885 & $\begin{array}{l}\text { \# Daily Telegraph } \\
\text { (Launceston); South } \\
\text { Australian Chronicle; Sydney } \\
\text { Mail }\end{array}$ & 1886 \\
\hline$\%$ Ouida & "Don Gesualdo" & 1885 & South Australian Chronicle & 1886 \\
\hline $\begin{array}{l}\text { \% David } \\
\text { Christie } \\
\text { Murray }\end{array}$ & “The Queen's Scarf” & 1885 & Week & 1887 \\
\hline $\begin{array}{l}\text { Margaret } \\
\text { Oliphant }\end{array}$ & $\begin{array}{l}\text { "The Son of His } \\
\text { Father" }\end{array}$ & 1886 & $\begin{array}{l}\text { Adelaide Observer; Evening } \\
\text { Journal; Sydney Mail }\end{array}$ & 1886 \\
\hline $\begin{array}{l}\text { Lady M. } \\
\text { Majendie }\end{array}$ & "On the Scent" & 1886 & Telegraph; Week & 1887 \\
\hline William Black & "Sabina Zembra" & 1886 & $\begin{array}{l}\text { Adelaide Observer; Bendigo } \\
\text { Advertiser }\end{array}$ & 1887 \\
\hline M. E. Braddon & $\begin{array}{l}\text { "The One Thing } \\
\text { Needful" }\end{array}$ & 1886 & Age & 1886 \\
\hline $\begin{array}{l}\text { \% Annie S. } \\
\text { Swan }\end{array}$ & "Twice Tried" & 1886 & $\begin{array}{l}\text { Gippsland Times } \\
\text { Bendigo Advertiser; South } \\
\text { Australian Chronicle }\end{array}$ & $\begin{array}{l}1888 \\
1889\end{array}$ \\
\hline$\%$ Ouida & "A House Party" & 1886 & South Australian Chronicle & 1886 \\
\hline M. E. Braddon & "Like and Unlike" & 1887 & $\begin{array}{l}\text { South Australian Chronicle; } \\
\text { Provincial Syndicate } 4\end{array}$ & 1887 \\
\hline Walter Besant & "Herr Paulus" & 1887 & Age & 1887 \\
\hline $\begin{array}{l}\text { \% John Winter } \\
\text { Strange } \\
\text { (Stannard) }\end{array}$ & $\begin{array}{l}\text { "Beautiful Jim of the } \\
\text { Blankshire Regiment" }\end{array}$ & 1887 & Telegraph; Week & 1888 \\
\hline $\begin{array}{l}\text { \% Robert } \\
\text { Buchanan }\end{array}$ & "The Heir of Linne" & 1887 & Telegraph & 1889 \\
\hline Bret Harte & $\begin{array}{l}\text { "The Argonauts of } \\
\text { North Liberty" }\end{array}$ & 1888 & $\begin{array}{l}\text { Adelaide Observer; } \\
\text { Capricornian; Evening } \\
\text { Journal; Morning Bulletin; } \\
\text { Telegraph; Week }\end{array}$ & 1888 \\
\hline Wilkie Collins & "The Legacy of Cain" & 1888 & $\begin{array}{l}\text { South Australian Chronicle; } \\
\text { West Australian Times }\end{array}$ & 1888 \\
\hline$\%$ Emile Zola & "The Dream" & n.d. & West Australian Times & 1888 \\
\hline $\begin{array}{l}\text { H. Rider } \\
\text { Haggard }\end{array}$ & $\begin{array}{l}\text { "Colonel Quaritch, V. } \\
\text { C." }\end{array}$ & 1888 & $\begin{array}{l}\text { Adelaide Observer; Evening } \\
\text { Journal } \\
\text { Week }\end{array}$ & $\begin{array}{l}1888 \\
1889\end{array}$ \\
\hline $\begin{array}{l}\text { S. Baring } \\
\text { Gould }\end{array}$ & $\begin{array}{l}\text { "The } \\
\text { Pennycomequicks" }\end{array}$ & 1889 & $\begin{array}{l}\text { \# Bendigo Advertiser; Leader; } \\
\text { South Australian Chronicle }\end{array}$ & 1889 \\
\hline Hall Caine & "The Bondman" & 1889 & $\begin{array}{l}\text { Adelaide Observer; North } \\
\text { Melbourne Advertiser; } \\
\text { Telegraph; Provincial } \\
\text { Syndicate } 4\end{array}$ & 1889 \\
\hline $\begin{array}{l}\text { J. Fitzgerald } \\
\text { Molloy }\end{array}$ & $\begin{array}{l}\text { "How Came He } \\
\text { Dead?" }\end{array}$ & 1889 & $\begin{array}{l}\text { Week } \\
\text { Gippsland Times }\end{array}$ & $\begin{array}{l}1889 \\
1891\end{array}$ \\
\hline William Black & $\begin{array}{l}\text { "Stand Fast, Craig } \\
\text { Royston!" }\end{array}$ & 1890 & $\begin{array}{l}\text { Adelaide Observer; Age; } \\
\text { Week } \\
\text { North Queensland Register }\end{array}$ & $\begin{array}{r}1890 \\
1892 \\
\end{array}$ \\
\hline J. Monk Foster & "Slaves of Fate" & 1890 & Adelaide Observer; Evening & 1891 \\
\hline
\end{tabular}




\begin{tabular}{|c|c|c|c|c|}
\hline & & & $\begin{array}{l}\text { Journal; Telegraph } \\
\text { Oakleigh Leader }\end{array}$ & 1892 \\
\hline $\begin{array}{l}\text { \$ Margaret } \\
\text { Oliphant }\end{array}$ & $\begin{array}{l}\text { "The Heir Presumptive } \\
\text { and the Heir Apparent" }\end{array}$ & 1890 & $\begin{array}{l}\text { Capricornian; Morning } \\
\text { Bulletin } \\
\text { Age }\end{array}$ & $\begin{array}{l}1890 \\
1891\end{array}$ \\
\hline $\begin{array}{l}\text { \% Sir Gilbert } \\
\text { Campbell }\end{array}$ & "The Avenging Hand" & 1890 & $\begin{array}{l}\text { Adelaide Observer; Evening } \\
\text { Journal }\end{array}$ & 1891 \\
\hline \% Lily Tinsley & "A Living Lie" & 1890 & Telegraph & 1892 \\
\hline $\begin{array}{l}\text { \% Arthur } \\
\text { Marchmont }\end{array}$ & $\begin{array}{l}\text { "Miser Hoadley's } \\
\text { Secret" }\end{array}$ & 1890 & $\begin{array}{l}\text { Maitland Mercury; Telegraph } \\
\text { Gippsland Times }\end{array}$ & $\begin{array}{l}1892 \\
1894\end{array}$ \\
\hline J. Monk Foster & "A Miner’s Million" & 1891 & $\begin{array}{l}\text { Adelaide Observer; Evening } \\
\text { Journal; Telegraph }\end{array}$ & 1888 \\
\hline $\begin{array}{l}\text { \$ Robert } \\
\text { Buchanan }\end{array}$ & "The Wedding Ring" & 1891 & South Australian Chronicle & 1891 \\
\hline \$ James Payn & $\begin{array}{l}\text { "A Modern Dick } \\
\text { Whittington" }\end{array}$ & 1891 & $\begin{array}{l}\text { \# Queenslander; South } \\
\text { Australian Chronicle }\end{array}$ & 1892 \\
\hline J. Monk Foster & "A Crimson Fortune" & 1892 & Adelaide Observer & 1892 \\
\hline $\begin{array}{l}\text { Margaret } \\
\text { Oliphant }\end{array}$ & "The Sorceress" & 1892 & $\begin{array}{l}\text { \# Leader, Queenslander; } \\
\text { South Australian Chronicle }\end{array}$ & 1892 \\
\hline $\begin{array}{l}\text { \$ H. Rider } \\
\text { Haggard }\end{array}$ & "Nada the Lilly" & 1892 & $\begin{array}{l}\text { Bendigo Advertiser; Clarence } \\
\text { and Richmond Examiner; } \\
\text { West Australian Times }\end{array}$ & 1892 \\
\hline $\begin{array}{l}\text { \% Helen } \\
\text { Mathers }\end{array}$ & "My Other Self" & 1892 & Western Mail & 1894 \\
\hline $\begin{array}{l}\text { \% Marie } \\
\text { Carelli }\end{array}$ & "The Song of Miriam" & 1892 & Telegraph & 1893 \\
\hline M. E. Braddon & "The Venetians" & 1893 & $\begin{array}{l}\text { South Australian Chronicle } \\
\text { Bendigo Advertiser }\end{array}$ & $\begin{array}{l}1892 \\
1893 \\
\end{array}$ \\
\hline $\begin{array}{l}\text { \$ Thomas } \\
\text { Hardy }\end{array}$ & $\begin{array}{l}\text { "The Pursuit of the } \\
\text { Well-Beloved" }\end{array}$ & 1892 & $\begin{array}{l}\text { Leader; South Australian } \\
\text { Chronicle }\end{array}$ & 1892 \\
\hline $\begin{array}{l}\text { \$ David } \\
\text { Christie } \\
\text { Murray }\end{array}$ & $\begin{array}{l}\text { "Bob Martin's Little } \\
\text { Girl" }\end{array}$ & 1892 & $\begin{array}{l}\text { Australian Town and Country } \\
\text { Journal; Evening News; } \\
\text { Leader; South Australian } \\
\text { Chronicle; Telegraph; Week }\end{array}$ & 1892 \\
\hline $\begin{array}{l}\text { \$ W. Clark } \\
\text { Russell }\end{array}$ & $\begin{array}{l}\text { "Alone on a Wide, } \\
\text { Wide Sea" }\end{array}$ & 1892 & $\begin{array}{l}\text { Adelaide Observer; } \\
\text { Capricornian; Evening } \\
\text { Journal; Leader; Morning } \\
\text { Bulletin }\end{array}$ & 1891 \\
\hline $\begin{array}{l}\text { William Henry } \\
\text { Moyes }\end{array}$ & $\begin{array}{l}\text { "The Mormon's } \\
\text { Daughter" }\end{array}$ & 1893 & $\begin{array}{l}\text { Clarence and Richmond } \\
\text { Examiner }\end{array}$ & 1893 \\
\hline $\begin{array}{l}\text { Margaret } \\
\text { Oliphant }\end{array}$ & $\begin{array}{l}\text { "That House in } \\
\text { Bloomsbury" }\end{array}$ & 1893 & $\begin{array}{l}\text { Age; South Australian } \\
\text { Chronicle }\end{array}$ & 1893 \\
\hline Mary Albert & $\begin{array}{l}\text { "The Luckiest Man in } \\
\text { the World" }\end{array}$ & 1893 & Daily Telegraph (Launceston) & 1894 \\
\hline $\begin{array}{l}\text { \$ William } \\
\text { Black }\end{array}$ & "Highland Cousins" & 1893 & Sydney Mail & 1894 \\
\hline $\begin{array}{l}\text { \$ M. E. } \\
\text { Braddon }\end{array}$ & "All Along the River" & 1893 & $\begin{array}{l}\text { \# Leader; South Australian } \\
\text { Chronicle; Telegraph; Week; } \\
\text { West Australian Times }\end{array}$ & 1893 \\
\hline $\begin{array}{l}\text { \$ H. Rider } \\
\text { Haggard }\end{array}$ & $\begin{array}{l}\text { "Montezuma's } \\
\text { Daughter" }\end{array}$ & 1893 & Sydney Mail & 1893 \\
\hline $\begin{array}{l}\text { Adeline } \\
\text { Sergeant }\end{array}$ & "Marjorie's Mistake" & 1894 & $\begin{array}{l}\text { Bendigo Advertiser } \\
\text { Barrier Miner }\end{array}$ & $\begin{array}{l}1894 \\
1895 \\
\end{array}$ \\
\hline $\begin{array}{l}\text { J. Fitzgerald } \\
\text { Molloy }\end{array}$ & "On Wheels of Fire" & 1894 & $\begin{array}{l}\text { Elsternwick Leader; Oakleigh } \\
\text { Leader }\end{array}$ & 1893 \\
\hline J. Monk Foster & "Judith Saxon" & 1894 & $\begin{array}{l}\text { South Australian Chronicle } \\
\text { Brisbane Courier }\end{array}$ & $\begin{array}{l}1894 \\
1895\end{array}$ \\
\hline $\begin{array}{l}\text { \$ H. Rider } \\
\text { Haggard }\end{array}$ & $\begin{array}{l}\text { "The People of the } \\
\text { Mist" }\end{array}$ & 1894 & Argus & 1894 \\
\hline
\end{tabular}




\begin{tabular}{|c|c|c|c|c|}
\hline $\begin{array}{l}\% \text { M. E. } \\
\text { Braddon }\end{array}$ & "Thou Art the Man" & 1894 & $\begin{array}{l}\text { Adelaide Observer; Age; } \\
\text { Telegraph; Warwick Argus; } \\
\text { Week }\end{array}$ & 1894 \\
\hline $\begin{array}{l}\text { A. W. } \\
\text { Marchmont }\end{array}$ & "Sir Jaffray’s Wife" & 1895 & Bendigo Advertiser & 1895 \\
\hline $\begin{array}{l}\text { Ernest } \\
\text { Glanville }\end{array}$ & "The Golden Rock" & 1895 & Telegraph & 1895 \\
\hline Dora Russell & "The Drift of Fate" & 1895 & $\begin{array}{l}\text { Age; South Australian } \\
\text { Chronicle; Telegraph }\end{array}$ & 1895 \\
\hline $\begin{array}{l}\text { \% M. E. } \\
\text { Braddon }\end{array}$ & "Sons of Fire" & 1895 & $\begin{array}{l}\text { Adelaide Observer; Evening } \\
\text { Journal; Leader; Week }\end{array}$ & 1895 \\
\hline John K. Leys & "The Broken Fetter" & 1896 & Telegraph; Week & 1896 \\
\hline $\begin{array}{l}\text { \$ M. E. } \\
\text { Braddon }\end{array}$ & $\begin{array}{l}\text { "When the World Was } \\
\text { Younger" }\end{array}$ & 1895 & South Australian Chronicle & 1896 \\
\hline $\begin{array}{l}\text { \$ Mrs } \\
\text { Hungerford }\end{array}$ & $\begin{array}{l}\text { "The Professor's } \\
\text { Experiment" }\end{array}$ & 1895 & Telegraph & 1896 \\
\hline $\begin{array}{l}\text { John Winter } \\
\text { Strange }\end{array}$ & $\begin{array}{l}\text { "The Colonel's } \\
\text { Daughter" }\end{array}$ & 1896 & Week & 1896 \\
\hline G. A. Henty & “The Queen's Cup" & 1896 & $\begin{array}{l}\text { Adelaide Observer; } \\
\text { Telegraph; Week }\end{array}$ & 1893 \\
\hline R. J. Charlton & The Honourable Jim" & 1896 & Bendigo Advertiser & 1897 \\
\hline M. E. Braddon & "The Little Auntie" & 1896 & Week & 1897 \\
\hline $\begin{array}{l}\text { Florence } \\
\text { Marryat }\end{array}$ & $\begin{array}{l}\text { "In the Name of } \\
\text { Liberty" }\end{array}$ & 1897 & $\begin{array}{l}\text { Adelaide Observer; } \\
\text { Telegraph; Week }\end{array}$ & 1897 \\
\hline $\begin{array}{l}\text { \$ Walter } \\
\text { Besant }\end{array}$ & The Master Craftsman" & 1896 & Age & 1896 \\
\hline $\begin{array}{l}\text { Mary H. } \\
\text { Tennyson }\end{array}$ & "Within Her Grasp" & 1897 & Adelaide Observer; Telegraph & 1896 \\
\hline J. Monk Foster & $\begin{array}{l}\text { "The Watchman of } \\
\text { Orsden Moss" }\end{array}$ & 1897 & $\begin{array}{l}\text { Adelaide Observer; Bendigo } \\
\text { Advertiser; Capricornian; } \\
\text { Morning Bulletin }\end{array}$ & 1897 \\
\hline $\begin{array}{l}\text { Ernest } \\
\text { Glanville }\end{array}$ & “The Lover's Quest” & 1897 & Week & 1897 \\
\hline $\begin{array}{l}\text { A. W. } \\
\text { Marchmont }\end{array}$ & "In the Grip of Hate" & 1898 & $\begin{array}{l}\text { Capricornian; Daily } \\
\text { Telegraph (Launceston); } \\
\text { Morning Bulletin }\end{array}$ & 1898 \\
\hline E. W. Hornung & "Young Blood" & 1898 & Leader & 1897 \\
\hline $\begin{array}{l}\text { William Le } \\
\text { Queux }\end{array}$ & "The Bond of Black" & 1898 & Leader; Telegraph; Week & 1899 \\
\hline $\begin{array}{l}\text { Ernest } \\
\text { Glanville }\end{array}$ & $\begin{array}{l}\text { "His Enemy's } \\
\text { Daughter" }\end{array}$ & 1898 & Launceston Examiner & 1898 \\
\hline William Black & "Wild Eelin" & 1898 & $\begin{array}{l}\text { Age; Australian Town and } \\
\text { Country Journal; Brisbane } \\
\text { Courier; South Australian } \\
\text { Chronicle }\end{array}$ & 1898 \\
\hline $\begin{array}{l}\text { Iza Duffus } \\
\text { Hardy }\end{array}$ & $\begin{array}{l}\text { "MacGilleroy's } \\
\text { Millions" }\end{array}$ & 1899 & $\begin{array}{l}\text { Adelaide Observer; Daily } \\
\text { Telegraph (Launceston) }\end{array}$ & 1899 \\
\hline Walter Besant & $\begin{array}{l}\text { "The Fourth } \\
\text { Generation" }\end{array}$ & 1899 & Age & 1899 \\
\hline
\end{tabular}

